\title{
MOREAU-YOSIDA REGULARIZATION IN STATE CONSTRAINED ELLIPTIC CONTROL PROBLEMS: ERROR ESTIMATES AND PARAMETER ADJUSTMENT*
}

\author{
MICHAEL HINTERMÜLLER ${ }^{\dagger}$ AND MICHAEL HINZE ${ }^{\ddagger}$
}

\begin{abstract}
An adjustment scheme for the regularization parameter of a Moreau-Yosida-based regularization, or relaxation, approach to the numerical solution of pointwise state constrained elliptic optimal control problems is introduced. The method utilizes error estimates of an associated finite element discretization of the regularized problems for the optimal selection of the regularization parameter in dependence on the mesh size of discretization and error estimates for the approximation error due to regularization. The theoretical results are verified numerically.
\end{abstract}

Key words. elliptic optimal control problem, error estimates, Moreau-Yosida-based regularization, pointwise state constraints

AMS subject classifications. 49M15, 49M37, 65K05, 65N12, 65N30, $90 \mathrm{C} 33$

DOI. $10.1137 / 080718735$

1. Introduction. In this paper we are interested in the numerical analysis of a Moreau-Yosida-based regularization of PDE-constrained optimization problems subject to pointwise state constraints. This regularization scheme along with a primaldual path-following algorithm was considered in [9] in order to approximate the measure-valued Lagrange multiplier of the original state constrained problem by a sequence of regular multipliers. Advantages of such a regularization procedure are the availability of highly efficient solvers for the regularized problem (such as semismooth Newton methods) and the induced numerical stability with respect to the mesh size of discretization $[8,10]$. This latter property, however, relies on an appropriate tuning of the regularization parameter. Suitable adjustment strategies link the regularization parameter to the mesh size in order to balance the regularization and discretization errors properly. As such parameter selection rules are currently not available for the type of regularization considered here, the aim of this work is to close this gap and, thus, to allow for fine tuned implementations of corresponding solution algorithms.

In order to set up a model problem class for our subsequent discussion, let $\Omega \subset$ $\mathbb{R}^{d}(d=2,3)$ be a bounded domain with a smooth boundary $\partial \Omega$ and consider the second order linear elliptic differential operator

$$
A y:=-\sum_{i, j=1}^{d} \partial_{x_{j}}\left(a_{i j} y_{x_{i}}\right)+\sum_{i=1}^{d} b_{i} y_{x_{i}}+c y
$$

where, for simplicity, the coefficients $a_{i j}, b_{i}$, and $c$ are assumed to be smooth functions in $\bar{\Omega}$. Let $a(\cdot, \cdot)$ denote the bilinear form associated with the differential operator $A$.

*Received by the editors March 17, 2008; accepted for publication (in revised form) December 26, 2008; published electronically April 22, 2009.

http://www.siam.org/journals/sinum/47-3/71873.html

$\dagger$ Department of Mathematics, University of Sussex, Falmer, Brighton, UK, and Institute for Mathematics and Scientific Computing, University of Graz, Graz, Austria (hint@mathematik.huberlin.de). This author gratefully acknowledges support by the FWF under START Program Y305 "Interfaces and Free Boundaries."

${ }^{\ddagger}$ Schwerpunkt Optimierung und Approximation, Universität Hamburg, Bundesstraße 55, 20146 Hamburg, Germany (michael.hinz@uni-hamburg.de). This author gratefully acknowledges the support of the DFG Priority Program 1253 entitled Optimization With Partial Differential Equations.

1666 
For some constant $c_{1}>0$, it is assumed to satisfy

$$
a(v, v) \geq c_{1}\|v\|_{H^{1}}^{2} \quad \text { for all } v \in H^{1}(\Omega) .
$$

Next, let $f \in\left(H^{1}(\Omega)\right)^{\prime}$. Then it follows that the elliptic boundary value problem

$$
\begin{aligned}
A y & =f & & \text { in } \Omega, \\
\sum_{i, j=1}^{d} a_{i j} y_{x_{i}} \nu_{j} & =0 & & \text { on } \partial \Omega
\end{aligned}
$$

admits a unique solution $y \in H^{1}(\Omega)$, which we denote by $y=\mathcal{G} f$. Here, $\nu$ is the outward unit normal to $\partial \Omega$. Furthermore, if $f \in L^{2}(\Omega)$, then the solution $y$ belongs to $H^{2}(\Omega)$ and satisfies $\|y\|_{H^{2}} \leq C\|f\|_{L^{2}}$.

The Hilbert space of controls is denoted by $\left(U,(\cdot, \cdot)_{U}\right)$ and is identified with its dual. Further, $B: U \rightarrow L^{2}(\Omega)$ is a linear, continuous operator which models the impact of the control action. Subsequently, we study the following model problem class:

$$
\begin{aligned}
\operatorname{minimize} J(w) & =\frac{1}{2} \int_{\Omega}\left|\mathcal{G} B w-y_{0}\right|^{2}+\frac{\alpha}{2}\|w\|_{U}^{2} \quad \text { over } \quad w \in U \\
\text { subject to } \mathcal{G} B w & \leq b \text { a.e. in } \Omega,
\end{aligned}
$$

where $\alpha>0, y_{0} \in H^{1}(\Omega)$, and $b \in W^{2, \infty}(\Omega)$ are given. Here and throughout, "a.e." stands for almost everywhere. We also invoke the following constraint qualification (Slater condition):

$$
\text { There exist } \hat{u} \in U, \tau>0: \quad \mathcal{G} B \hat{u} \leq b-\tau \text { a.e. in } \Omega .
$$

If $B$ is invertible, then our model problem satisfies the Slater condition automatically.

Standard techniques guarantee that problem (1.3) admits a unique solution $u \in U$. Moreover, from [3, Theorem 2] we deduce that there exist unique functions $\lambda \in \mathcal{M}(\bar{\Omega})$ and $p \in L^{2}(\Omega)$ satisfying, together with $y=\mathcal{G} B u$, the dual system

$$
\begin{gathered}
\int_{\Omega} p A v=\int_{\Omega}\left(y-y_{0}\right) v+\int_{\bar{\Omega}} v d \lambda \quad \forall v \in H^{2}(\Omega) \text { with } \sum_{i, j=1}^{d} a_{i j} v_{x_{i}} \nu_{j}=0 \text { on } \partial \Omega \\
B^{*} p+\alpha u=0 \quad \text { in } U, \\
\lambda \geq 0, y \leq b \text { a.e. in } \Omega \text { and } \int_{\bar{\Omega}}(b-y) d \lambda=0 .
\end{gathered}
$$

Here, $\mathcal{M}(\bar{\Omega})$ denotes the space of Radon measures, which is defined as the dual space of $\mathcal{C}(\bar{\Omega})$, and $B^{*}$ is the adjoint of $B$. The space $\mathcal{M}(\bar{\Omega})$ is endowed with the norm

$$
\|\lambda\|_{\mathcal{M}(\bar{\Omega})}=\sup _{f \in \mathcal{C}(\bar{\Omega}),|f| \leq 1} \int_{\bar{\Omega}} f d \lambda .
$$

Subsequently, we also use $\langle\lambda, b-y\rangle_{\mathcal{M}(\bar{\Omega}), \mathcal{C}(\bar{\Omega})}$ instead of $\int_{\bar{\Omega}}(b-y) d \lambda$.

A finite element analysis of problem (1.3) was carried out in [6] (compare also [5]) yielding the error bounds

$$
\left\|u-u_{h}\right\|_{U},\left\|y-y_{h}\right\|_{H^{1}}= \begin{cases}O\left(h^{\frac{1}{2}}\right) & \text { if } d=2, \\ O\left(h^{\frac{1}{4}}\right) & \text { if } d=3,\end{cases}
$$

Copyright $@$ by SIAM. Unauthorized reproduction of this article is prohibited. 
where $u_{h}$ denotes the variational discrete control (see [11]) and $y_{h}$ the associated piecewise linear and continuous discrete state. If, in addition, $B u \in W^{1, s}(\Omega)$, then

$$
\left\|u-u_{h}\right\|_{U}, \quad\left\|y-y_{h}\right\|_{H^{1}} \leq C h^{\frac{3}{2}-\frac{d}{2 s}} \sqrt{|\log h|} .
$$

As noted earlier, our aim is to investigate a finite element approximation of a Moreau-Yosida-based regularization (or, alternatively, augmented Lagrangian-type penalization) technique for the numerical solution of (1.3) and to provide suitable adjustment strategies for the regularization/penalization parameter with respect to a given finite element mesh. We emphasize that our subsequent results readily carry over, e.g., to the case of Dirichlet (rather than Neumann) boundary conditions. From here onwards and without loss of generality, it is convenient to set $b \equiv 0$.

The Moreau-Yosida-regularized version of (1.3) reads

$$
\min _{w \in U} J(w)=\frac{1}{2} \int_{\Omega}\left|\mathcal{G} B w-y_{0}\right|^{2}+\frac{\alpha}{2}\|w\|_{U}^{2}+\frac{1}{2 \gamma} \int_{\Omega}|\max (0, \bar{\lambda}+\gamma \mathcal{G} B w)|^{2},
$$

where $\gamma>0$ denotes the regularization (or penalization) parameter and $\bar{\lambda} \geq 0$, with $\bar{\lambda} \in L^{2}(\Omega)$, a fixed shift parameter, which represents an approximation of the Lagrange multiplier associated with the constraint $y \leq 0$. Thus, (1.9) resembles an augmented Lagrangian treatment of problem (1.3); see [8,9]. The problem (1.9) admits a unique solution $u^{\gamma} \in U$; see [9]. Furthermore, there exists a unique $p^{\gamma} \in H^{2}(\Omega)$ satisfying the adjoint system

$$
\begin{aligned}
a\left(v, p^{\gamma}\right)=\int_{\Omega}\left(y^{\gamma}-y_{0}\right) v+\int_{\Omega}\left(\bar{\lambda}+\gamma y^{\gamma}\right)^{+} v \quad \forall v & \in H^{1}(\Omega), \\
B^{*} p^{\gamma}+\alpha u^{\gamma} & =0 \text { in } U,
\end{aligned}
$$

where $y^{\gamma}=\mathcal{G} B u^{\gamma}$ and $(\cdot)^{+}=\max (0, \cdot)$ in the pointwise sense. If $\bar{\lambda} \equiv 0$ and $y^{\gamma}$ is feasible for (1.3), then $y^{\gamma}=y$ and $u^{\gamma}=u$. From our subsequent results we conclude that, given a mesh size of discretization $h$, there is an upper bound for $\gamma$ at which the error due to regularization is of the order of the discretization error. Increasing $\gamma$ beyond this $h$-dependent threshold does not improve the overall approximation error and, as (1.9) is harder to solve the larger $\gamma$ becomes, would result in unnecessary extra work in the solution procedure.

The rest of the paper is organized as follows: In section 2 we investigate the dependence of problem (1.9) on the parameter $\gamma$. For the finite element analysis developed in this work we provide uniform bounds with respect to $\gamma$ on $\left|y^{\gamma}\right|_{2}$ and $\frac{1}{\gamma}\left|p^{\gamma}\right|_{2}$, and we prove the decay rate

$$
\left\|\left(y^{\gamma}\right)^{+}\right\|_{L^{2}} \leq C \omega\left(\gamma^{-1}\right) \gamma^{-1 / 2}
$$

with $\omega(z) \downarrow 0$ for $z \downarrow 0$. This is used in Lemma 3.1 to provide the $h$-dependent bound

$$
\left\|\left(y^{\gamma}\right)^{+}\right\|_{\mathcal{C}(\bar{\Omega})} \leq C\left(h^{1-\frac{d}{q}}+\gamma^{-\frac{1}{2}} h^{-\frac{d}{2}}\right),
$$

which is used in (2.12) to obtain the error estimate

$$
\left\|y^{\gamma}-y\right\|_{H^{1}}+\left\|u^{\gamma}-u\right\|_{U} \leq C\left\|u^{\gamma}-u\right\|_{U} \leq C\left(h^{1-\frac{d}{q}}+\gamma^{-\frac{1}{2}} h^{-\frac{d}{2}}\right)^{\frac{1}{2}}+\frac{C}{\sqrt{\gamma}}\|\bar{\lambda}\|_{L^{2}},
$$


where $y$ and $u$ denote the solutions of (1.3) and $0<h \leq 1$ is arbitrary. Here and below, we use the seminorm $|y|_{2}=\left\|A_{0} y\right\|_{L^{2}}$, where $A_{0}$ denotes the leading part of the differential operator $A$, i.e.,

$$
A_{0}=-\sum_{i, j=1}^{d} \partial_{x_{j}}\left(a_{i j} y_{x_{i}}\right) .
$$

In section 3 we present the finite element analysis of problem (1.9). Among other aspects we prove the error bounds

$$
\left\|y^{\gamma}-y_{h}^{\gamma}\right\|_{H^{1}}+\left\|u^{\gamma}-u_{h}^{\gamma}\right\|_{U} \leq C\left(h+\gamma h^{2}\right)
$$

and also the following estimate which is uniform with respect to $\gamma$ :

$$
\left\|y^{\gamma}-y_{h}^{\gamma}\right\|_{H^{1}}+\left\|u^{\gamma}-u_{h}^{\gamma}\right\|_{U} \leq C h^{1-\frac{d}{4}} .
$$

Here and below, $y_{h}^{\gamma}$ and $u_{h}^{\gamma}$ denote the finite element approximations to $y^{\gamma}$ and $u^{\gamma}$, respectively. We note that the latter estimate is in the spirit of (1.8). For the overall errors we finally obtain the main result of the present work which is proved in Theorem 3.7:

$$
\begin{aligned}
\left\|y-y_{h}^{\gamma}\right\|_{H^{1}}^{2}+\left\|u-u_{h}^{\gamma}\right\|_{U}^{2} \sim & \left\|y-y^{\gamma}\right\|_{H^{1}}^{2}+\left\|u-u^{\gamma}\right\|_{U}^{2}+\left\|y^{\gamma}-y_{h}^{\gamma}\right\|_{H^{1}}^{2}+\left\|u^{\gamma}-u_{h}^{\gamma}\right\|_{U}^{2} \\
& \leq C\left(\max \left(h^{1-\frac{d}{q}}, h^{2-\frac{d}{2}}\right)+\gamma^{-\frac{1}{2}} h^{-\frac{d}{2}}+\gamma^{-1}\|\bar{\lambda}\|_{L^{2}}^{2}\right) .
\end{aligned}
$$

Based on this estimate we in section 4 propose strategies for adjusting $\gamma$ to $h$ and present numerical results which confirm our theoretical findings.

We point out that error estimates for the Moreau-Yosida regularization of pointwise state constraints are currently not available in the literature. In that respect, the present contribution closes this gap and allows a numerically fine-tuned implementation of the primal-dual path-following algorithm in [9].

Error estimates for alternative regularization schemes can be found in $[12,13]$. In contrast to these strategies, the Moreau-Yosida-based approach does not rely on a pointwise regularization of the state constraint; rather it relaxes the original problem by using a penalized $L^{2}$-averaging of the constraint violation.

We note that all subsequent considerations carry over to bilateral constraints of the form $a \leq y \leq b$ with sufficiently regular bounds $a$ and $b$ satisfying $b-a \geq \epsilon>0$ in $\bar{\Omega}$.

2. Analysis of the Moreau-Yosida-regularized problem (1.9). For our error analysis in the subsequent section we need several results concerning the boundedness and convergence behavior of $\left(y^{\gamma}, u^{\gamma}, p^{\gamma}\right)$ as $\gamma \rightarrow \infty$. Throughout, we use the notation

$$
J^{\gamma}(v)=J(v)+\frac{1}{2 \gamma} \int_{\Omega}\left((\bar{\lambda}+\gamma \mathcal{G} B v)^{+}\right)^{2}
$$

We start by observing that for $\gamma \geq 1$

$$
J\left(u^{\gamma}\right) \leq J^{\gamma}\left(u^{\gamma}\right) \leq J^{\gamma}(u) \leq J(u)+\frac{1}{2 \gamma}\|\bar{\lambda}\|_{L^{2}}^{2} \leq J(u)+\frac{1}{2}\|\bar{\lambda}\|_{L^{2}}^{2}=: C_{u} .
$$

Hence, $\frac{1}{2 \gamma} \int_{\Omega}\left(\left(\bar{\lambda}+\gamma \mathcal{G} B u^{\gamma}\right)^{+}\right)^{2}$ is uniformly bounded. Moreover, in [9, Proposition 2.1] it was shown that $u^{\gamma} \rightarrow u$ strongly in $L^{2}(\Omega)$. Hence, (2.1) implies

$$
\left\|\left(y^{\gamma}\right)^{+}\right\|_{L^{2}}=\omega\left(\gamma^{-1}\right) \gamma^{-1 / 2}
$$

Copyright $@$ by SIAM. Unauthorized reproduction of this article is prohibited. 
with

$$
\omega\left(\gamma^{-1}\right)=2 \max \left(\frac{1}{\gamma}\|\bar{\lambda}\|_{L^{2}}^{2},\left(J(u)-J\left(u^{\gamma}\right)\right)^{+}\right)^{1 / 2}
$$

which satisfies $\omega(z) \downarrow 0$ as $z \downarrow 0$. Moreover, (2.1) yields

$$
\max \left(\left\|y^{\gamma}\right\|_{L^{2}}^{2}, \alpha\left\|u^{\gamma}\right\|_{U}^{2}\right) \leq 2 C_{u}
$$

Since $y^{\gamma}=\mathcal{G} B u^{\gamma}$ for all $\gamma$, from elliptic regularity, (2.3), and $\|B u\|_{L^{2}} \leq C_{B}$ for some constant $C_{B} \geq 0$ independent of $\gamma$, we infer

$$
\left|y^{\gamma}\right|_{2}=\left\|A_{0} y^{\gamma}\right\|_{L^{2}} \leq C_{0}
$$

with some positive constant $C_{0}$ independent of $\gamma$. For the adjoint state $p^{\gamma}$ we obtain

$$
\left|p^{\gamma}\right|_{2} \leq \sqrt{2 C_{u}}\left(1+\omega\left(\gamma^{-1}\right) \sqrt{\gamma}\right)+\left\|y_{0}\right\|_{L^{2}} \leq C_{0}^{*}\left(1+\omega\left(\gamma^{-1}\right) \sqrt{\gamma}\right)
$$

with a positive constant $C_{0}^{*}$ independent of $\gamma$. In fact, for (2.5) we use the adjoint equation (1.10) together with (2.2) and (2.3).

Next, we estimate the distance between $(y, u)$ and $\left(y^{\gamma}, u^{\gamma}\right)$.

THEOREM 2.1. Let $u$ denote the solution of (1.3) and $u^{\gamma}$ the solution of (1.9). Then we have

$$
\alpha\left\|u-u^{\gamma}\right\|_{U}^{2}+\left\|y-y^{\gamma}\right\|_{L^{2}}^{2}+\gamma\left\|\left(y^{\gamma}\right)^{+}\right\|_{L^{2}}^{2} \leq \frac{1}{\gamma}\|\bar{\lambda}\|_{L^{2}}^{2}+\left\langle\lambda, y^{\gamma}\right\rangle_{\mathcal{M}(\bar{\Omega}), \mathcal{C}(\bar{\Omega})},
$$

and for the feasibility violation there holds

$$
\left\|\left(y^{\gamma}\right)^{+}\right\|_{L^{2}} \leq \sqrt{\frac{2}{\gamma}} \max \left(\frac{\|\bar{\lambda}\|_{L^{2}}^{2}}{\gamma},\left|\left\langle\lambda,\left(y^{\gamma}\right)^{+}\right\rangle_{\mathcal{M}(\bar{\Omega}), \mathcal{C}(\bar{\Omega})}\right|\right)^{1 / 2} .
$$

Proof. The first order optimality systems for the original and the Moreau-Yosidaregularized problem yield

$$
\alpha\left(u-u^{\gamma}\right)=B^{*}\left(p^{\gamma}-p\right) .
$$

Multiplying by $u-u^{\gamma}$ and using the respective first order system we get

$$
\begin{aligned}
\alpha\left\|u-u^{\gamma}\right\|_{U}^{2} & =\int_{\Omega} B\left(u-u^{\gamma}\right)\left(p^{\gamma}-p\right)=a\left(y-y^{\gamma}, p^{\gamma}-p\right) \\
& =-\left\|y-y^{\gamma}\right\|_{L^{2}}^{2}+\left\langle\lambda, y^{\gamma}\right\rangle_{\mathcal{M}(\bar{\Omega}), \mathcal{C}(\bar{\Omega})}+\int_{\Omega}\left(\bar{\lambda}+\gamma y^{\gamma}\right)^{+}\left(y-y^{\gamma}\right) \\
& =-\left\|y-y^{\gamma}\right\|_{L^{2}}^{2}+\left\langle\lambda, y^{\gamma}\right\rangle_{\mathcal{M}(\bar{\Omega}), \mathcal{C}(\bar{\Omega})}-\gamma\left\|\left(y^{\gamma}\right)^{+}\right\|_{L^{2}\left(\Omega_{\gamma}^{+}(\bar{\lambda})\right)}^{2}+\int_{\Omega_{\gamma}^{+}(\bar{\lambda})} \bar{\lambda} y^{\gamma},
\end{aligned}
$$

where $\Omega_{\gamma}^{+}(\bar{\lambda}):=\left\{\bar{\lambda}+\gamma y^{\gamma}>0\right\}$. Next, we observe

$$
-y^{\gamma}<\frac{\bar{\lambda}}{\gamma} \text { a.e. in } \Omega_{\gamma}^{+}(\bar{\lambda}) .
$$

Due to $\bar{\lambda} \geq 0$ a.e. in $\Omega$, we further note that

$$
\Omega_{\gamma}^{+}(\bar{\lambda}) \supseteq \Omega_{\gamma}^{+}(0)=\left\{y^{\gamma}>0\right\} .
$$

Copyright (c) by SIAM. Unauthorized reproduction of this article is prohibited. 
Using (2.9) and (2.10) we continue (2.8) and obtain

$$
\alpha\left\|u-u^{\gamma}\right\|_{U}^{2}+\left\|y-y^{\gamma}\right\|_{L^{2}}^{2}+\gamma\left\|\left(y^{\gamma}\right)^{+}\right\|_{L^{2}}^{2} \leq \frac{1}{\gamma}\|\bar{\lambda}\|_{L^{2}}^{2}+\left\langle\lambda, y^{\gamma}\right\rangle_{\mathcal{M}(\bar{\Omega}), \mathcal{C}(\bar{\Omega})} .
$$

Next, recall that $y^{\gamma} \in \mathcal{C}(\bar{\Omega})$. Hence, we also have $\left(y^{\gamma}\right)^{+} \in \mathcal{C}(\bar{\Omega})$. Together with $\lambda \in \mathcal{M}(\bar{\Omega})$ and $\lambda \geq 0$ we further estimate (2.11):

$$
\alpha\left\|u-u^{\gamma}\right\|_{U}^{2}+\left\|y-y^{\gamma}\right\|_{L^{2}}^{2}+\gamma\left\|\left(y^{\gamma}\right)^{+}\right\|_{L^{2}}^{2} \leq \frac{1}{\gamma}\|\bar{\lambda}\|_{L^{2}}^{2}+\left\langle\lambda,\left(y^{\gamma}\right)^{+}\right\rangle_{\mathcal{M}(\bar{\Omega}), \mathcal{C}(\bar{\Omega})} .
$$

From this we obtain

$$
\left\|\left(y^{\gamma}\right)^{+}\right\|_{L^{2}} \leq \sqrt{\frac{2}{\gamma}} \max \left(\frac{\|\bar{\lambda}\|_{L^{2}}^{2}}{\gamma},\left|\left\langle\lambda,\left(y^{\gamma}\right)^{+}\right\rangle_{\mathcal{M}(\bar{\Omega}), \mathcal{C}(\bar{\Omega})}\right|\right)^{1 / 2},
$$

which proves the assertion.

We end this section by studying sufficient conditions for

$$
\left\|\left(y^{\gamma}\right)^{+}\right\|_{L^{2}}=\mathcal{O}\left(\gamma^{-1}\right) \text { as } \gamma \rightarrow \infty .
$$

THEOREM 2.2. Let $u$ denote the solution of (1.3) and $u^{\gamma}$ the solution of (1.9). If $y_{0} \geq 0$ a.e. in $\Omega$, then $\left\|\left(y^{\gamma}\right)^{+}\right\|_{L^{2}}=\mathcal{O}\left(\gamma^{-1}\right)$ as $\gamma \rightarrow \infty$.

Proof. The optimal solution $\left(u^{\gamma}, y^{\gamma}=\mathcal{G B} u^{\gamma}\right)$ together with the corresponding adjoint state satisfy

$$
\begin{aligned}
& a\left(v, p^{\gamma}\right)=\int_{\Omega}\left(y^{\gamma}-y_{0}\right) v+\int_{\Omega}\left(\bar{\lambda}+\gamma y^{\gamma}\right)^{+} v \quad \forall v \in H^{1}(\Omega), \\
& a\left(y^{\gamma}, w\right)=\int_{\Omega} B u^{\gamma} w \quad \forall w \in H^{1}(\Omega) .
\end{aligned}
$$

Using $v=y^{\gamma}$ in the first and $w=p^{\gamma}$ in the second equation, respectively, together with (1.11) and subtracting yield

$$
0=\left\|y^{\gamma}\right\|_{L^{2}}^{2}-\int_{\Omega} y_{0} y^{\gamma}+\int_{\Omega_{+}^{\gamma}(\bar{\lambda})} \bar{\lambda} y^{\gamma}+\gamma \int_{\Omega_{+}^{\gamma}(\bar{\lambda})}\left(y^{\gamma}\right)^{2}+\alpha\left\|u^{\gamma}\right\|_{U}^{2} .
$$

Now, our assumption $y_{0} \geq 0$ yields

$$
\gamma\left\|\left(y^{\gamma}\right)^{+}\right\|_{L^{2}}^{2} \leq \int_{\Omega} y_{0} y^{\gamma}+\gamma^{-1} \int_{\Omega_{+}^{\gamma}(\bar{\lambda}) \backslash \Omega_{+}(0)} \bar{\lambda}^{2} \leq \int_{\Omega} y_{0}\left(y^{\gamma}\right)^{+}+\gamma^{-1} \int_{\Omega_{+}^{\gamma}(\bar{\lambda}) \backslash \Omega_{+}(0)} \bar{\lambda}^{2} .
$$

From this we conclude

$$
\left\|\left(y^{\gamma}\right)^{+}\right\|_{L^{2}}=\mathcal{O}\left(\gamma^{-1}\right) \text { as } \gamma \rightarrow \infty .
$$

In view of the proof of Theorem 2.2 we also have the following result. By $w^{-}$we denote the negative part of $w$, i.e., $w=w^{+}-w^{-}$.

TheOREM 2.3. Let $u$ denote the solution of (1.3) and $u^{\gamma}$ the solution of (1.9). If there exists $\epsilon>0$ such that

$$
-\int_{\Omega} y_{0}^{-} y-\|y\|_{L^{2}}^{2}-\alpha\|u\|_{U}^{2} \leq-\epsilon
$$

then $\left\|\left(y^{\gamma}\right)^{+}\right\|_{L^{2}}=\mathcal{O}\left(\gamma^{-1}\right)$ as $\gamma \rightarrow \infty$.

Copyright $@$ by SIAM. Unauthorized reproduction of this article is prohibited. 
Proof. Similar to in [9] we get $u^{\gamma} \rightarrow u$ strongly in $L^{2}(\Omega)$ and $y^{\gamma} \rightarrow y$ strongly in $H^{1}(\Omega)$. Hence, for sufficiently large $\gamma$, we conclude

$$
-\int_{\Omega} y_{0}^{-} y^{\gamma}-\left\|y^{\gamma}\right\|_{L^{2}}^{2}-\alpha\left\|u^{\gamma}\right\|_{U}^{2} \leq-\frac{\epsilon}{2}<0
$$

Then (2.15) yields

$$
\gamma\left\|\left(y^{\gamma}\right)^{+}\right\|_{L^{2}}^{2} \leq \int_{\Omega} y_{0}\left(y^{\gamma}\right)^{+}+\gamma^{-1} \int_{\Omega_{+}^{\gamma}(\bar{\lambda}) \backslash \Omega_{+}(0)} \bar{\lambda}^{2} .
$$

From this we conclude

$$
\left\|\left(y^{\gamma}\right)^{+}\right\|_{L^{2}}=\mathcal{O}\left(\gamma^{-1}\right) \text { as } \gamma \rightarrow \infty .
$$

3. Finite element discretization and error analysis of (1.9). Let $\mathcal{T}_{h}$ be a triangulation of $\Omega$ with maximum mesh size $h:=\max _{T \in \mathcal{T}_{h}} \operatorname{diam}(T)$ and vertices $x_{1}, \ldots, x_{m}$, where $\operatorname{diam}(T)$ denotes the diameter of $T$. We suppose that $\bar{\Omega}$ is the union of the elements of $\mathcal{T}_{h}$ so that element edges lying on the boundary are curved. In addition, we assume that the triangulation is quasi-uniform in the sense that there exists a constant $\kappa>0$ (independent of $h$ ) such that each $T \in \mathcal{T}_{h}$ is contained in a ball of radius $\kappa^{-1} h$ and contains a ball of radius $\kappa h$. Let us define the space of linear finite elements

$$
X_{h}:=\left\{v_{h} \in \mathcal{C}(\bar{\Omega}) \mid v_{h} \text { is a linear polynomial on each } T \in \mathcal{T}_{h}\right\}
$$

with the appropriate modification for boundary elements. We use the following approximation and inverse properties:

(a) According to [4] combined with [2, Theorem 4.4.20] the $L^{2}$-projection $\Pi_{h}$ : $W^{1, q}(\Omega) \rightarrow X_{h}(d<q \leq \infty)$ satisfies

$$
\left\|v-\Pi_{h} v\right\|_{L^{\infty}} \leq C h^{1-\frac{d}{q}}\|v\|_{W^{1, q}}
$$

and is stable in $L^{2}$.

(b) Due to [2, Lemma 4.5.3], for $v_{h} \in X_{h}$ we have

$$
\left\|v_{h}\right\|_{L^{\infty}} \leq C h^{-\frac{d}{2}}\left\|v_{h}\right\|_{L^{2}}
$$

In what follows it is convenient to introduce a discrete approximation of the operator $\mathcal{G}$. In fact, for a given function $v \in L^{2}(\Omega)$ we denote by $z_{h}=\mathcal{G}_{h} v \in X_{h}$ the solution of the discrete Neumann problem

$$
a\left(z_{h}, v_{h}\right)=\int_{\Omega} v v_{h} \quad \text { for all } v_{h} \in X_{h} .
$$

It is well-known [14] that for all $v \in L^{2}(\Omega)$

$$
\begin{aligned}
\left\|\mathcal{G} v-\mathcal{G}_{h} v\right\|_{L^{2}} & \leq C h^{2}\|v\|_{L^{2}}, \\
\left\|\mathcal{G} v-\mathcal{G}_{h} v\right\|_{L^{\infty}} & \leq C h^{2-\frac{d}{2}}\|v\|_{L^{2}} .
\end{aligned}
$$

The estimate (3.4) can be improved provided one strengthens the assumption on $v$. 
3.1. Estimate for $\left\|\boldsymbol{y}^{\gamma}-\boldsymbol{y}\right\|_{\boldsymbol{H}^{1}}+\left\|\boldsymbol{u}^{\gamma}-\boldsymbol{u}\right\|_{\boldsymbol{U}}$. We recall that by (2.12)

$$
\alpha\left\|u-u^{\gamma}\right\|_{U}^{2}+\left\|y-y^{\gamma}\right\|_{L^{2}}^{2}+\gamma\left\|\left(y^{\gamma}\right)^{+}\right\|_{L^{2}}^{2} \leq \frac{1}{\gamma}\|\bar{\lambda}\|_{L^{2}}^{2}+\left\langle\lambda,\left(y^{\gamma}\right)^{+}\right\rangle_{\mathcal{M}(\bar{\Omega}), \mathcal{C}(\bar{\Omega})} .
$$

Since the right-hand side of this estimate is uniformly bounded in $\gamma$, we obtain $\left\|\left(y^{\gamma}\right)^{+}\right\|_{L^{2}}=\mathcal{O}\left(\gamma^{-\frac{1}{2}}\right)$ as $\gamma \rightarrow \infty$. The decay of $\left\|y^{\gamma}-y\right\|_{L^{2}}^{2}+\alpha\left\|u^{\gamma}-u\right\|_{U}^{2}$ with respect to $\gamma$ can be estimated by that of $\left\|\left(y^{\gamma}\right)^{+}\right\|_{\mathcal{C}(\bar{\Omega})}$. To the best of the authors' knowledge, an estimate of the latter term has not yet been obtained. As a partial result in this direction we have the following lemma which bridges the gap between the averaged $L^{2}$-type penalization of the constraint violation and the required uniform bound for $\left(y^{\gamma}\right)^{+}$.

Lemma 3.1. Let $0<h \leq 1$. Then, for $q \in(d, \infty)$ for $d=2$, and $q \in(d, 6)$ for $d=3$ we have

$$
\left\|\left(y^{\gamma}\right)^{+}\right\|_{\mathcal{C}(\bar{\Omega})} \leq C\left(h^{1-\frac{d}{q}}+\gamma^{-\frac{1}{2}} h^{-\frac{d}{2}}\right),
$$

where the positive constant $C$ is independent of $\gamma$ and $h$.

Proof. We use (3.1), (3.2), and (2.2) to obtain

$$
\begin{aligned}
\left\|\left(y^{\gamma}\right)^{+}\right\|_{\mathcal{C}(\bar{\Omega})} & \leq\left\|\left(y^{\gamma}\right)^{+}-\Pi_{h}\left(y^{\gamma}\right)^{+}\right\|_{\mathcal{C}(\bar{\Omega})}+\left\|\Pi_{h}\left(y^{\gamma}\right)^{+}\right\|_{\mathcal{C}(\bar{\Omega})} \\
& \leq C\left\{h^{1-\frac{d}{q}}\left\|\left(y^{\gamma}\right)^{+}\right\|_{1, q}+h^{-\frac{d}{2}}\left\|\Pi_{h}\left(y^{\gamma}\right)^{+}\right\|_{L^{2}}\right\} \\
& \leq C\left\{h^{1-\frac{d}{q}}\left\|u^{\gamma}\right\|_{U}+h^{-\frac{d}{2}}\left\|\left(y^{\gamma}\right)^{+}\right\|_{L^{2}}\right\} \\
& \leq C\left(h^{1-\frac{d}{q}}+\gamma^{-\frac{1}{2}} h^{-\frac{d}{2}}\right) .
\end{aligned}
$$

This concludes the proof.

From

$c_{1}\left\|y-y^{\gamma}\right\|_{H^{1}}^{2} \leq a\left(y-y^{\gamma}, y-y^{\gamma}\right)=\int_{\Omega} B\left(u-u^{\gamma}\right)\left(y-y^{\gamma}\right) \leq C\left\|u-u^{\gamma}\right\|_{U}\left\|y^{\gamma}-y\right\|_{H^{1}}$ we immediately infer

$$
\left\|y^{\gamma}-y\right\|_{H^{1}}+\left\|u^{\gamma}-u\right\|_{U} \leq C\left\|u^{\gamma}-u\right\|_{U} \leq \frac{C}{\sqrt{\alpha}}\left(h^{1-\frac{d}{q}}+\gamma^{-\frac{1}{2}} h^{-\frac{d}{2}}\right)^{\frac{1}{2}}+\frac{C}{\sqrt{\alpha \gamma}}\|\bar{\lambda}\|_{L^{2}} .
$$

Under additional regularity assumptions, the bound in Lemma 3.1 also holds for $q=\infty$, i.e.,

$$
\left\|\left(y^{\gamma}\right)^{+}\right\|_{\mathcal{C}(\bar{\Omega})} \leq C\left(h+\gamma^{-\frac{1}{2}} h^{-\frac{d}{2}}\right) .
$$

In this case, (3.6) becomes

$$
\left\|y^{\gamma}-y\right\|_{H^{1}}+\left\|u^{\gamma}-u\right\|_{U} \leq C\left\|u^{\gamma}-u\right\|_{U} \leq \frac{C}{\sqrt{\alpha}}\left(h+\gamma^{-\frac{1}{2}} h^{-\frac{d}{2}}\right)^{\frac{1}{2}}+\frac{C}{\sqrt{\alpha \gamma}}\|\bar{\lambda}\|_{L^{2}} .
$$

Remark 3.2. If the assumptions of Theorems 2.2 or 2.3 hold true, then the estimate (3.6) improves:

Copyright (c) by SIAM. Unauthorized reproduction of this article is prohibited. 


$$
\left\|y^{\gamma}-y\right\|_{H^{1}}+\left\|u^{\gamma}-u\right\|_{U} \leq C\left\|u^{\gamma}-u\right\|_{U} \leq \frac{C}{\sqrt{\alpha}}\left(h^{1-\frac{d}{q}}+\gamma^{-1} h^{-\frac{d}{2}}\right)^{\frac{1}{2}}+\frac{C}{\sqrt{\alpha \gamma}}\|\bar{\lambda}\|_{L^{2}} .
$$

Similarly, instead of (3.8) we obtain

$$
\left\|y^{\gamma}-y\right\|_{H^{1}}+\left\|u^{\gamma}-u\right\|_{U} \leq C\left\|u^{\gamma}-u\right\|_{U} \leq \frac{C}{\sqrt{\alpha}}\left(h+\gamma^{-1} h^{-\frac{d}{2}}\right)^{\frac{1}{2}}+\frac{C}{\sqrt{\alpha \gamma}}\|\bar{\lambda}\|_{L^{2}} .
$$

3.2. Estimate for $\left\|\boldsymbol{y}^{\gamma}-\boldsymbol{y}_{\boldsymbol{h}}^{\gamma}\right\|_{\boldsymbol{H}^{1}}+\left\|\boldsymbol{u}^{\gamma}-\boldsymbol{u}_{\boldsymbol{h}}^{\gamma}\right\|_{\boldsymbol{U}}$. Problem (1.9) is now approximated by the following sequence of control problems depending on the mesh parameter $h$ :

$$
\min _{u \in U} J_{h}(u):=\frac{1}{2} \int_{\Omega}\left|\mathcal{G}_{h} B u-y_{0}\right|^{2}+\frac{\alpha}{2}\|u\|_{U}^{2}+\frac{1}{2} \int_{\Omega}\left|\left(\bar{\lambda}+\gamma \mathcal{G}_{h} B u\right)^{+}\right|^{2} .
$$

Problem (3.11) represents a convex infinite-dimensional optimization problem of a structure similar to problem (1.9). It admits a unique solution $u_{h}^{\gamma}$ with corresponding state $y_{h}^{\gamma} \in X_{h}$. Furthermore, there exists a unique function $p_{h}^{\gamma} \in X_{h}$ satisfying

$$
\begin{array}{r}
a\left(v_{h}, p_{h}^{\gamma}\right)=\int_{\Omega}\left(y_{h}^{\gamma}-y_{0}+\left(\bar{\lambda}+\gamma y_{h}^{\gamma}\right)^{+}\right) v_{h} \text { for all } v_{h} \in X_{h} \text { and } \\
\alpha u_{h}^{\gamma}+B^{*} p_{h}^{\gamma}=0 \text { in } U .
\end{array}
$$

Let us first prove an error estimate which is optimal with respect to the approximation order of the finite element space, but it depends on the relaxation parameter $\gamma$.

THEOREM 3.3. Let $u^{\gamma}$ denote the solution of (1.9) with $y^{\gamma}=\mathcal{G B} u^{\gamma}$, and $u_{h}^{\gamma}$ the solution of (3.11). Then there exist $h_{0}>0$ and a constant $C$ independent of $\gamma$ and $h$ such that

$$
\left\|u^{\gamma}-u_{h}^{\gamma}\right\|_{U} \leq \frac{C}{\alpha} \gamma h^{2} \text { for all } 0<h \leq h_{0}
$$

Proof. Let $y^{h}, p^{h}, p^{h h} \in X_{h}$ denote the solutions to

$$
\begin{aligned}
a\left(y^{h}, v_{h}\right) & =\left(B u^{\gamma}, v_{h}\right) \text { for all } v_{h} \in X_{h}, \\
a\left(v_{h}, p^{h}\right) & =\int_{\Omega}\left(y^{\gamma}-y_{0}+\left(\bar{\lambda}+\gamma y^{\gamma}\right)^{+}\right) v_{h} \text { for all } v_{h} \in X_{h}, \\
a\left(v_{h}, p^{h h}\right) & =\int_{\Omega}\left(y^{h}-y_{0}+\left(\bar{\lambda}+\gamma y^{h}\right)^{+}\right) v_{h} \text { for all } v_{h} \in X_{h} .
\end{aligned}
$$

Subtracting (1.11) from (3.13) yields

$$
\begin{aligned}
\alpha\left\|u^{\gamma}-u_{h}^{\gamma}\right\|_{U}^{2}= & \int_{\Omega}\left(p_{h}^{\gamma}-p^{\gamma}\right) B\left(u^{\gamma}-u_{h}^{\gamma}\right) \\
= & \int_{\Omega}\left(p^{\gamma}-p^{h}\right) B\left(u_{h}^{\gamma}-u^{\gamma}\right)+\int_{\Omega}\left(p^{h}-p^{h h}\right) B\left(u_{h}^{\gamma}-u^{\gamma}\right) \\
& +\int_{\Omega}\left(p^{h h}-p_{h}^{\gamma}\right) B\left(u_{h}^{\gamma}-u^{\gamma}\right) \\
= & (1)+(2)+(3) .
\end{aligned}
$$

Copyright (c) by SIAM. Unauthorized reproduction of this article is prohibited. 
Since $p^{\gamma} \in H^{2}(\Omega)$, it is straightforward to show

$$
(1) \leq C\left\|p^{\gamma}-p^{h}\right\|_{L^{2}}\left\|u^{\gamma}-u_{h}^{\gamma}\right\|_{U} \leq C h^{2}\left|p^{\gamma}\right|_{2}\left\|u^{\gamma}-u_{h}^{\gamma}\right\|_{U} .
$$

Furthermore,

$$
\begin{aligned}
(2) & =a\left(y_{h}^{\gamma}-y^{h}, p^{h}-p^{h h}\right) \\
& =\int_{\Omega}\left(y^{\gamma}-y^{h}\right)\left(y_{h}^{\gamma}-y^{h}\right)+\left(\left(\bar{\lambda}+\gamma y^{\gamma}\right)^{+}-\left(\bar{\lambda}+\gamma y^{h}\right)^{+}\right)\left(y_{h}^{\gamma}-y^{h}\right) \\
& \leq C(1+\gamma)\left\|y^{\gamma}-y^{h}\right\|_{L^{2}}\left\|u^{\gamma}-u_{h}^{\gamma}\right\|_{U} \leq C h^{2}(1+\gamma)\left|y^{\gamma}\right|_{2}\left\|u^{\gamma}-u_{h}^{\gamma}\right\|_{U}
\end{aligned}
$$

and

$$
\begin{aligned}
(3) & =a\left(y_{h}^{\gamma}-y^{h}, p^{h h}-p_{h}^{\gamma}\right) \\
& =\int_{\Omega}\left(y^{h}-y_{h}^{\gamma}\right)\left(y_{h}^{\gamma}-y^{h}\right)+\overbrace{\left(\left(\bar{\lambda}+\gamma y^{h}\right)^{+}-\left(\bar{\lambda}+\gamma y_{h}^{\gamma}\right)^{+}\right)\left(y_{h}^{\gamma}-y^{h}\right)}^{\leq 0 \text { by Lemma } A .1} \leq 0
\end{aligned}
$$

such that

$$
\left\|u^{\gamma}-u_{h}^{\gamma}\right\|_{U} \leq \frac{C}{\alpha} h^{2}\left\{(1+\gamma)\left|y^{\gamma}\right|_{2}+\left|p^{\gamma}\right|_{2}\right\}
$$

Using the bounds (2.5) and (2.4) on $\left|p^{\gamma}\right|_{2}$ and $\left|y^{\gamma}\right|_{2}$, respectively, proves the assertion.

Since

$$
\left\|y^{\gamma}-y_{h}^{\gamma}\right\|_{H^{1}} \leq\left\|y^{\gamma}-y^{h}\right\|_{H^{1}}+\left\|y^{h}-y_{h}^{\gamma}\right\|_{H^{1}} \leq C\left(h\left\|u_{\gamma}\right\|_{U}+\left\|u^{\gamma}-u_{h}^{\gamma}\right\|_{U}\right)
$$

by the Lipschitz continuity of $\left\|y^{h}-y_{h}^{\gamma}\right\|_{H^{1}}$ with respect to $u$, we obtain with the help of $(3.14)$

$$
\left\|y^{\gamma}-y_{h}^{\gamma}\right\|_{H^{1}}+\left\|u^{\gamma}-u_{h}^{\gamma}\right\|_{U} \leq C\left(h+\frac{\gamma}{\alpha} h^{2}\right) .
$$

Next, we prove an error estimate in $h$ which is independent of $\gamma$. For this purpose we first prove a uniform (in $\gamma) L^{1}$-bound for $\left(\bar{\lambda}+\gamma y^{\gamma}\right)^{+}$and $\left(\bar{\lambda}+\gamma y_{h}^{\gamma}\right)^{+}$.

Lemma 3.4. Let assumption 1.4 be satisfied, and let $u^{\gamma}$ and $u_{h}^{\gamma}$ denote the unique solutions to (1.9) and (3.11), respectively, with associated states $y^{\gamma}$ and $y_{h}^{\gamma}$. Then

$$
\left\|\max \left(\left(\bar{\lambda}+\gamma y^{\gamma}\right)^{+},\left(\bar{\lambda}+\gamma y_{h}^{\gamma}\right)^{+}\right)\right\|_{L^{1}(\Omega)} \leq C
$$

with some positive constant $C$ independent of $\gamma$ and of $h$.

Proof. Using $\hat{u}$ of assumption 1.4 as a test function in (1.11) yields

$$
\alpha\left(u^{\gamma}, \hat{u}\right)+\left(B^{*} \mathcal{G}^{*}\left(\mathcal{G} B u^{\gamma}-y_{0}\right), \hat{u}\right)=0,
$$

where the action of the operator $\mathcal{G}^{*}$ is defined in (2.14). Thus,

$$
\int_{\Omega} \tau\left(\bar{\lambda}+\gamma y^{\gamma}\right)^{+} d x \leq-\int_{\Omega}\left(\bar{\lambda}+\gamma y^{\gamma}\right)^{+} \mathcal{G} B \hat{u} d x=\alpha\left(u^{\gamma}, \hat{u}\right)+\int_{\Omega}\left(y^{\gamma}-y_{0}\right) \mathcal{G} B \hat{u} d x \leq C
$$

independent of $\gamma$ due to the continuity of $B$ and $\mathcal{G}$ and the uniform bounds on $\left\|u^{\gamma}\right\|_{U}$ with respect to $\gamma$. This proves the claim for $\left\|\left(\bar{\lambda}+\gamma y^{\gamma}\right)^{+}\right\|_{L^{1}(\Omega)}$. Since 
$\left\|\mathcal{G}_{h} B \hat{u}-\mathcal{G} B \hat{u}\right\|_{L^{\infty}} \rightarrow 0$ for $h \rightarrow 0$ by (3.4) and the solutions $u_{h}^{\gamma}$ to (3.11) are uniformly bounded in $U$ with respect to $\gamma$ and $h$, similar arguments give the desired estimate also for $\left\|\left(\bar{\lambda}+\gamma y_{h}^{\gamma}\right)^{+}\right\|_{L^{1}(\Omega)}$ with a possibly smaller constant $0<\tilde{\tau} \leq \tau$.

We are now prepared to prove a $\gamma$-independent error estimate.

THEOREM 3.5. Let $u^{\gamma}$ denote the solution of (1.9) with $y^{\gamma}=\mathcal{G B} u^{\gamma}$, and $u_{h}^{\gamma}$ the solution to (3.11) with $y_{h}^{\gamma}=\mathcal{G}_{h} B u_{h}^{\gamma}$. Then there exist $h_{0} \in(0,1]$ and a constant $C$ independent of $\gamma$ and $h$ such that

$$
\left\|u^{\gamma}-u_{h}^{\gamma}\right\|_{U}+\left\|y^{\gamma}-y_{h}^{\gamma}\right\|_{H^{1}} \leq C h^{1-\frac{d}{4}} \text { for all } 0<h \leq h_{0} .
$$

Proof. Let $y^{h}, p^{h} \in X_{h}$ denote the finite element approximations defined at the beginning of the proof of Theorem 3.3. Next, we multiply the difference of (1.11) and (3.13) by $u^{\gamma}-u_{h}^{\gamma}$. This gives

$$
\begin{aligned}
\alpha\left\|u^{\gamma}-u_{h}^{\gamma}\right\|_{U}^{2} & =\int_{\Omega}\left(p^{\gamma}-p_{h}^{\gamma}\right) B\left(u_{h}^{\gamma}-u^{\gamma}\right) d x \\
& =\int_{\Omega}\left(p^{\gamma}-p_{h}\right) B\left(u_{h}^{\gamma}-u^{\gamma}\right) d x+\int_{\Omega}\left(p^{h}-p_{h}^{\gamma}\right) B\left(u_{h}^{\gamma}-u^{\gamma}\right) d x=:(1)+(2) .
\end{aligned}
$$

We proceed by estimating

$$
\begin{aligned}
(1) & \leq \frac{\alpha}{2}\left\|u_{h}^{\gamma}-u^{\gamma}\right\|_{U}^{2}+\frac{C}{\alpha}\left\|p^{\gamma}-p^{h}\right\|_{L^{2}}^{2} \\
& \leq \frac{\alpha}{2}\left\|u_{h}^{\gamma}-u^{\gamma}\right\|_{U}^{2}+\frac{C}{\alpha} h^{4-d}\left(\left\|y^{\gamma}-y_{0}\right\|_{L^{2}}^{2}+\left\|\left(\bar{\lambda}+\gamma y^{\gamma}\right)^{+}\right\|_{L^{1}}^{2}\right),
\end{aligned}
$$

where we have used [1] to estimate the finite element error $\left\|p^{\gamma}-p^{h}\right\|_{L^{2}}$. Further, using the definition of the auxilliary functions $y^{h}$ and $p^{h}$ and the optimality conditions, we get

$$
\begin{aligned}
(2)= & a\left(y_{h}^{\gamma}-y^{h}, p^{h}-p_{h}^{\gamma}\right)=\int_{\Omega}\left(y^{\gamma}-y_{h}^{\gamma}\right)\left(y_{h}^{\gamma}-y^{h}\right) \\
& +\left(\left(\bar{\lambda}+\gamma y^{\gamma}\right)^{+}-\left(\bar{\lambda}+\gamma y_{h}^{\gamma}\right)^{+}\right)\left(y_{h}^{\gamma}-y^{h}\right) d x \\
= & -\left\|y^{\gamma}-y_{h}^{\gamma}\right\|_{L^{2}}^{2}+\int_{\Omega}\left(y^{\gamma}-y_{h}^{\gamma}\right)\left(y^{\gamma}-y^{h}\right) \\
& +\left(\left(\bar{\lambda}+\gamma y^{\gamma}\right)^{+}-\left(\bar{\lambda}+\gamma y_{h}^{\gamma}\right)^{+}\right)\left(y_{h}^{\gamma}-y^{h}\right) d x \\
\leq & -\frac{1}{2}\left\|y^{\gamma}-y_{h}^{\gamma}\right\|_{L^{2}}^{2}+\frac{1}{2}\left\|y^{\gamma}-y^{h}\right\|_{L^{2}}^{2}+\int_{\Omega}\left(\left(\bar{\lambda}+\gamma y^{\gamma}\right)^{+}-\left(\bar{\lambda}+\gamma y_{h}^{\gamma}\right)^{+}\right)\left(y_{h}^{\gamma}-y^{h}\right) d x \\
= & -\frac{1}{2}\left\|y^{\gamma}-y_{h}^{\gamma}\right\|_{L^{2}}^{2}+\frac{1}{2}\left\|y^{\gamma}-y^{h}\right\|_{L^{2}}^{2}+\underbrace{\int_{\Omega}\left(\left(\bar{\lambda}+\gamma y^{\gamma}\right)^{+}-\left(\bar{\lambda}+\gamma y_{h}^{\gamma}\right)^{+}\right)\left(y_{h}^{\gamma}-y^{\gamma}\right) d x}_{\Omega 0 \text { by Lemma } A .1} \\
& +\int_{\Omega}\left(\bar{\lambda}+\gamma y^{\gamma}\right)^{+}\left(y^{\gamma}-y^{h}\right) d x+\int_{\Omega}\left(\bar{\lambda}+\gamma y_{h}^{\gamma}\right)^{+}\left(y^{h}-y^{\gamma}\right) d x \\
\leq & -\frac{1}{2}\left\|y^{\gamma}-y_{h}^{\gamma}\right\|_{L^{2}}^{2}+\frac{1}{2}\left\|y^{\gamma}-y^{h}\right\|_{L^{2}}^{2} \\
& +\max \left(\left\|\left(\bar{\lambda}+\gamma y^{\gamma}\right)^{+}\right\|_{L^{1}},\left\|\left(\bar{\lambda}+\gamma y_{h}^{\gamma}\right)^{+}\right\|_{L^{1}}\right)\left\|y^{\gamma}-y^{h}\right\|_{L^{\infty}} .
\end{aligned}
$$

Copyright $@$ ㅇ by SIAM. Unauthorized reproduction of this article is prohibited. 
Combining (1) and (2) we obtain, with the help of (3.3) and (3.4),

$$
\begin{aligned}
\alpha\left\|u^{\gamma}-u_{h}^{\gamma}\right\|_{U}^{2}+\frac{1}{2}\left\|y^{\gamma}-y_{h}^{\gamma}\right\|_{L^{2}}^{2} & \\
\leq C & \left(\frac{1}{\alpha} h^{4-d}\left(\left\|y^{\gamma}-y_{0}\right\|_{L^{2}}^{2}+\left\|\left(\bar{\lambda}+\gamma y^{\gamma}\right)^{+}\right\|_{L^{1}}^{2}\right)+h^{2}\left\|u^{\gamma}\right\|_{U}^{2}\right. \\
& \left.\quad+h^{2-\frac{d}{2}} \max \left(\left\|\left(\bar{\lambda}+\gamma y^{\gamma}\right)^{+}\right\|_{L^{1}},\left\|\left(\bar{\lambda}+\gamma y_{h}^{\gamma}\right)^{+}\right\|_{L^{1}}\right)\right) .
\end{aligned}
$$

Using this estimate and again

$$
\left\|y^{\gamma}-y_{h}^{\gamma}\right\|_{H^{1}} \leq\left\|y^{\gamma}-y^{h}\right\|_{H^{1}}+\left\|y^{h}-y_{h}^{\gamma}\right\|_{H^{1}} \leq C\left\{h\left\|u_{\gamma}\right\|_{U}+\left\|u^{\gamma}-u_{h}^{\gamma}\right\|_{U}\right\},
$$

we finally get the desired result, since $h_{0} \leq 1$.

We have

$$
\left\|\mathcal{G} v-\mathcal{G}_{h} v\right\|_{L^{\infty}} \leq C\left\{\begin{array}{l}
h^{3-\frac{d}{s}}\|v\|_{W^{1, s}}, \\
h^{2}|\log h|^{2}\|v\|_{L^{\infty}},
\end{array}\right.
$$

where the first case of the right-hand side in (3.17) is due to [6, Lemma 2.1] for $v \in W^{1, s}(\Omega)$, with $1<s<\frac{d-1}{d}$, and the second case comes from [7, Lemma 1] with $v \in L^{\infty}(\Omega)$.

Now, let us assume that either $B u^{\gamma}$ is uniformly bounded in $W^{1, s}(\Omega)$ for some $1<s<\frac{d-1}{d}$, or $B u^{\gamma}$ is uniformly bounded in $L^{\infty}(\Omega)$. Then we deduce the following result from the proof of the previous theorem.

Corollary 3.6. Let the assumptions of Theorem 3.5 hold true. Then we have for all $0<h \leq h_{0}$

$$
\left\|u^{\gamma}-u_{h}^{\gamma}\right\|_{U}+\left\|y^{\gamma}-y_{h}^{\gamma}\right\|_{H^{1}} \leq C \begin{cases}h^{\frac{3}{2}-\frac{d}{2 s}} \sqrt{|\log h|} & \text { if } B u^{\gamma} \text { is uniformly bounded in } W^{1, s}(\Omega), \\ h|\log h| & \text { if } B u^{\gamma} \text { is uniformly bounded in } L^{\infty}(\Omega),\end{cases}
$$

where $C>0$ denotes some constant independent of $\gamma$ and $h$.

3.3. Estimate for $\left\|\boldsymbol{y}-\boldsymbol{y}_{\boldsymbol{h}}^{\gamma}\right\|_{H^{1}}+\left\|\boldsymbol{u}-\boldsymbol{u}_{\boldsymbol{h}}^{\gamma}\right\|_{U}$. The overall error allows the decomposition

$$
\left\|y-y_{h}^{\gamma}\right\|_{H^{1}}+\left\|u-u_{h}^{\gamma}\right\|_{U} \sim\left\|y-y^{\gamma}\right\|_{H^{1}}+\left\|u-u^{\gamma}\right\|_{U}+\left\|y^{\gamma}-y_{h}^{\gamma}\right\|_{H^{1}}+\left\|u^{\gamma}-u_{h}^{\gamma}\right\|_{U}
$$

so that the results of the previous sections may be combined to prove our main result.

THEOREM 3.7. Let $u$ and $u_{h}^{\gamma}$ denote the solutions of (1.3) and (3.11), respectively, with $y=\mathcal{G B u}$ and $y_{h}^{\gamma}=\mathcal{G}_{h} B u_{h}^{\gamma}$ the associated states. Then there exist $h_{0} \in(0,1]$ and a constant $C>0$ independent of $\gamma$ and $h$ such that

$$
\left\|y-y_{h}^{\gamma}\right\|_{H^{1}}+\left\|u-u_{h}^{\gamma}\right\|_{U} \leq C\left\{\frac{1}{\sqrt{\alpha}}\left(h^{1-\frac{d}{q}}+\gamma^{-\frac{1}{2}} h^{-\frac{d}{2}}\right)^{\frac{1}{2}}+\frac{1}{\sqrt{\alpha \gamma}}\|\bar{\lambda}\|_{L^{2}}+h^{1-\frac{d}{4}}\right\}
$$

holds for all $0<h \leq h_{0}$ with $y^{\gamma}=\mathcal{G B} u^{\gamma} \in W^{1, q}(\Omega)$ and $q \in(d, \infty)$ for $d=2$ and $q \in(d, 6)$ for $d=3$.

Proof. The overall error $\left\|y-y_{h}^{\gamma}\right\|_{H^{1}}+\left\|u-u_{h}^{\gamma}\right\|_{U}$ allows the decompostion (3.19), whose first two addends on the right-hand side can be estimated with the help of (3.6). To estimate the remaining addends we use (3.16) so that the result follows. 
From Remark 3.2 and Corollary 3.6 as well as from (2.11) and (3.18), we obtain the following corollary.

COROLlary 3.8.

(i) If the assumptions of Theorems 2.2 or 2.3 hold true, we have for all $0<h \leq h_{0}$ and for $q \in(d, \infty)$ for $d=2$, and $q \in(d, 6)$ for $d=3$

$$
\left\|u-u_{h}^{\gamma}\right\|_{U}+\left\|y-y_{h}^{\gamma}\right\|_{H^{1}} \leq C\left\{\frac{1}{\sqrt{\alpha}}\left(h^{1-\frac{d}{q}}+\gamma^{-1} h^{-\frac{d}{2}}\right)^{\frac{1}{2}}+\frac{1}{\sqrt{\alpha \gamma}}\|\bar{\lambda}\|_{L^{2}}+h^{1-\frac{d}{4}}\right\} .
$$

(ii) If the assumptions of Theorems 2.2 or 2.3 together with the assumptions of Theorem 3.5 hold true, we have for all $0<h \leq h_{0}$ and for $q \in(d, \infty)$ for $d=2$, and $q \in(d, 6)$ for $d=3$

$$
\begin{aligned}
& \left\|u-u_{h}^{\gamma}\right\|_{U}+\left\|y-y_{h}^{\gamma}\right\|_{H^{1}} \\
& \leq C\left\{\frac{1}{\sqrt{\alpha}}\left(h^{1-\frac{d}{q}}+\gamma^{-1} h^{-\frac{d}{2}}\right)^{\frac{1}{2}}+\frac{1}{\sqrt{\alpha \gamma}}\|\bar{\lambda}\|_{L^{2}}+h^{\frac{3}{2}-\frac{d}{2 s}} \sqrt{|\log h|}\right\}
\end{aligned}
$$

(iii) If $\left(B u^{\gamma}\right)_{\gamma}$ is bounded in $L^{\infty}(\Omega)$ and there exists a constant c (independent of $\gamma)$ such that $\left\|\left(y^{\gamma}\right)^{+}\right\|_{L^{\infty}} \leq c \gamma^{-1}$, then

$$
\left\|u-u_{h}^{\gamma}\right\|_{U}+\left\|y-y_{h}^{\gamma}\right\|_{H^{1}} \leq C\left\{\frac{1}{\sqrt{\alpha \gamma}}\left(1+\|\bar{\lambda}\|_{L^{2}}\right)+h|\log h|\right\} .
$$

We note that, under additional regularity assumptions and utilizing (3.7) and (3.10), the overall error (3.21) becomes

$$
\left\|u-u_{h}^{\gamma}\right\|_{U}+\left\|y-y_{h}^{\gamma}\right\|_{H^{1}} \leq C\left\{\frac{1}{\sqrt{\alpha}}\left(h+\gamma^{-1} h^{-\frac{d}{2}}\right)^{\frac{1}{2}}+\frac{1}{\sqrt{\alpha \gamma}}\|\bar{\lambda}\|_{L^{2}}+h^{1-\frac{d}{4}}\right\} .
$$

A similar observation holds true for (3.22).

In the next section we use the estimates (3.20) and (3.22) to propose strategies for adjusting the penalization parameter $\gamma$ to the finite element mesh size $h$.

4. Numerical verification and parameter selection. We end this paper by illustrating our theoretical findings by numerical results. In our examples below we use homogeneous Dirichlet (rather then Neumann) boundary conditions. As mentioned earlier, our theory covers this case as well. In all test runs, in order to compactify the computations, we used a mass lumping technique which preserves the approximation order. Throughout, we have $\Omega=(0,1)^{2}, U=L^{2}(\Omega), B=\mathrm{id}$, and $\bar{\lambda} \equiv 0$.

Example 1. Our first example is taken from [9]. The data are as follows: $y_{0}\left(x_{1}, x_{2}\right)=$ $10\left(\sin \left(2 \pi x_{1}\right)+x_{2}\right), b \equiv 0.01$, and $\alpha=0.1$. We first solve the problem on a very fine mesh in order to generate a reference solution which we then restrict onto the respective (coarser) mesh for computing the relevant error quantity.

Figure 4.1 depicts the convergence behavior of $\left\|\left(y_{h}^{\gamma}\right)^{+}\right\|_{L^{2}}$ and of $u_{h}^{\gamma}$ for various mesh sizes and $\gamma$-values, respectively. Clearly, we have $\left\|\left(y_{h}^{\gamma}\right)^{+}\right\|_{L^{2}}=\mathcal{O}\left(\gamma^{-1}\right)$. In fact, it turns out that the assumption of Theorem 2.3 is satisfied. Hence, under the regularity properties of this example, the estimate (3.24) is applicable. This gives

$$
\left\|u-u_{h}^{\gamma}\right\|_{U}^{2} \leq C\left(h+\gamma^{-1} h^{-1}+h\right) .
$$

Copyright $@$ by SIAM. Unauthorized reproduction of this article is prohibited. 

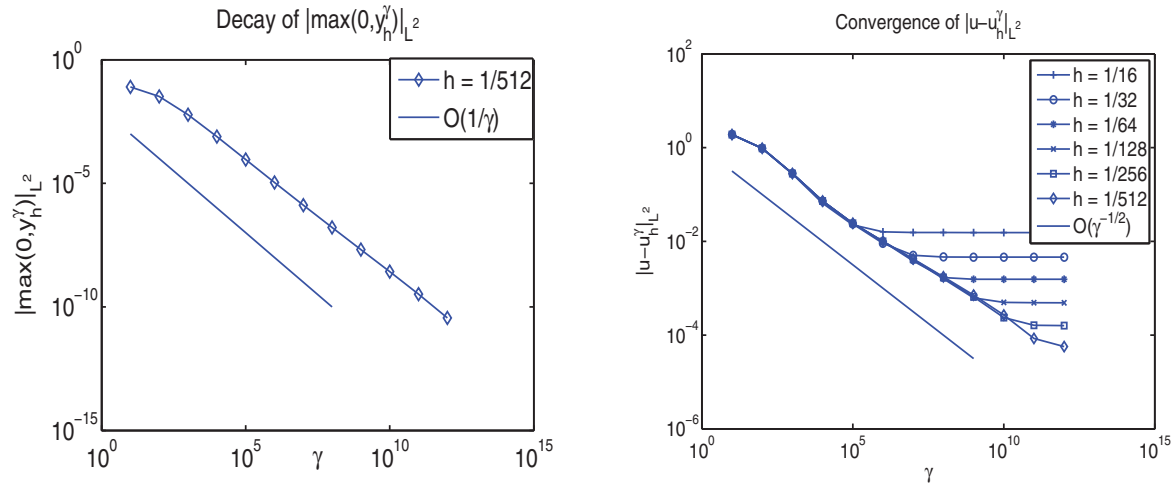

Fig. 4.1. Example 1. Decay of $\left\|\left(y_{h}^{\gamma}\right)^{+}\right\|_{L^{2}}$ (left) and the convergence of $\left\|u-u_{h}^{\gamma}\right\|_{U}$ (right).

Hence, $\gamma=h^{-2}$ is optimal as it produces an overall error of the order $\sqrt{h}$. Considering the bound on the right-hand side of (4.1) as a function of $\gamma$, we obtain

$$
\left\|u-u_{h}^{\gamma}\right\|_{U}=\mathcal{O}\left(\gamma^{-1 / 4}\right) .
$$

Our numerical results depicted in the right plot of Figure 4.1 indicate an even better rate of convergence at the order of $\mathcal{O}\left(\gamma^{-1 / 2}\right)$ (compare with the slope of the solid line). In fact, in our numerical tests we found that $\left\|\left(y_{h}^{\gamma}\right)^{+}\right\|_{L^{\infty}}=\mathcal{O}\left(\gamma^{-1}\right)$ which, in view of Corollary 3.8(iii), explains the improved rate for $\left(u_{h}^{\gamma}\right)_{\gamma}$. This shows that there are situations where the estimate of Lemma 3.1 is too pessimistic. The convergence results of Figure 4.1 further show that the $L^{2}$-error in $u_{h}^{\gamma}$ levels off as $\gamma$ increases. Also, the corresponding $\gamma$-threshold depends on the mesh size of discretization and is related to the point where the error due to relaxation equals the discretization error. Moreover, a reduction in $h$ results in an increase of the threshold as predicted by our theory.

Example 2. Our second example is constructed such that an explicit solution is available and such that the multiplier can be decomposed analytically into a regular part and a singular part concentrated on the boundary of the active set $\mathcal{A}:=$ $\{x \in \Omega: y(x)=0\}$ at the optimal solution. For later use we also define the corresponding inactive set $\mathcal{I}:=\Omega \backslash \mathcal{A}$. Moreover, this example violates the constraint qualification (1.4). Hence, the existence of $\lambda$ and $p$ cannot be argued by [3]. Rather one has to use weaker constraint qualifications. Such a theoretical investigation, however, is beyond the scope of the present work. Here, we study the numerical effect of such a situation only.

For the construction of the optimal state we define

$$
\hat{y}\left(x_{1}, x_{2}\right)=\sin \left(3 \pi x_{1}\right) \sin \left(x_{2}\right) \sin \left(x_{2}-1\right) \sin \left(1+2 \pi x_{1}+2 \pi x_{2}^{2}\right) .
$$

Then the optimal state is given by

$$
y\left(x_{1}, x_{2}\right):=-\max \left(0, \hat{y}\left(x_{1}, x_{2}\right)\right)^{4},
$$

and the corresponding optimal control is $u:=-\Delta y$. The associated adjoint state is $p:=-\alpha u$ with $\alpha=1 \mathrm{e}-3$. Further, we introduce $\hat{\lambda}:=\Delta p-y$. Note that, on $\partial \mathcal{A}, p$ admits only a generalized second derivative containing an element of $\partial(\hat{y})^{+}$, where we have used $\partial$ for representing the subdifferential of a convex function. Let $g^{\mathcal{A}}$ denote an arbitrarily fixed element of the generalized derivative at $\partial \mathcal{A}$ involved in $\Delta p$. Then 

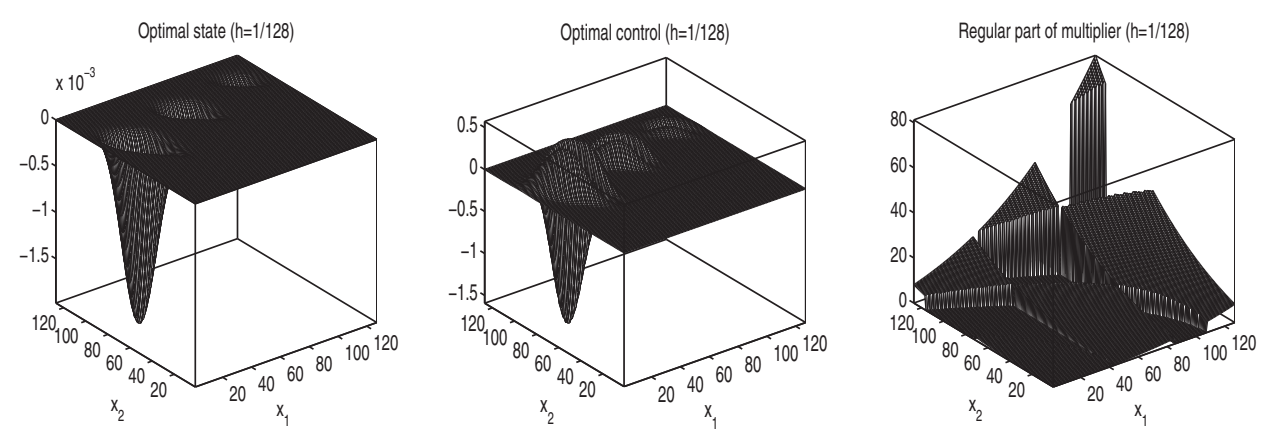

FIG. 4.2. Example 2. Optimal state (left), optimal control (middle), and the regular part of the associated multiplier (right).

one can show that $g^{\mathcal{A}}$ is nonnegative. This allows us to decompose $\hat{\lambda}$ into a regular part $\hat{\lambda}_{r}=\left.\hat{\lambda}\right|_{\text {int } \mathcal{A}}+\left.\hat{\lambda}\right|_{\mathcal{I}}$ and a singular part concentrated on $\partial \mathcal{A}$, i.e., $\lambda_{s}=g^{\mathcal{A}}$. For $\left.\lambda\right|_{\mathcal{I}}:=\left.\hat{\lambda}\right|_{\mathcal{I}}$ it can be shown that it vanishes as a consequence of the complementarity system (1.7). For the determination of $\left.\lambda\right|_{\operatorname{int} \mathcal{A}}$ we first define the desired state $y_{0}$ by

$$
\left.y_{0}\right|_{\operatorname{int} \mathcal{A}}=-2 \min \left(0,\left.\hat{\lambda}\right|_{\operatorname{int} \mathcal{A}}\right)+\left.f\right|_{\operatorname{int} \mathcal{A}}
$$

where $f\left(x_{1}, x_{2}\right):=0.001 \cdot\left(2+7.5\left(x_{1}+x_{2}\right)\right)^{4}$ and

$$
\left.y_{0}\right|_{\mathcal{I}}=\left.y\right|_{\mathcal{I}}-\left.(\Delta p)\right|_{\mathcal{I}}
$$

As the right-hand side in (4.2) is continuous, we define $\left.y_{0}\right|_{\partial \mathcal{A}}$ by continuous extension. Then $\left.\lambda\right|_{\operatorname{int} \mathcal{A}}$ is given by

$$
\left.\lambda\right|_{\operatorname{int} \mathcal{A}}=\left.(\Delta p)\right|_{\operatorname{int} \mathcal{A}}+\left.z\right|_{\operatorname{int} \mathcal{A}}-\left.y\right|_{\operatorname{int} \mathcal{A}}
$$

and

$$
\left.\lambda\right|_{\partial \mathcal{A}}=g^{\mathcal{A}} .
$$

In Figure 4.2 we show the optimal state (left), the optimal control (middle), and the regular part of the associated Lagrange multiplier (right) for $h=1 / 128$. Combing the plots of $y$ and $\lambda$ we find that the active set structure is rather involved.

Figure 4.3 depicts the convergence behavior of $\left\|\left(y_{h}^{\gamma}\right)^{+}\right\|_{L^{2}}$ and of $u_{h}^{\gamma}$ for various mesh sizes and $\gamma$-values, respectively. As for Example 1, we have $\left\|\left(y_{h}^{\gamma}\right)^{+}\right\|_{L^{2}}=\mathcal{O}\left(\gamma^{-1}\right)$. In fact, again it turns out that the assumption of Theorem 2.3 is satisfied and (4.1) is available yielding $\left\|u-u_{h}^{\gamma}\right\|_{U}=\mathcal{O}\left(\gamma^{-1 / 2}\right)$. All other conclusions are similar to the ones for Example 1. Hence, the lack of a Slater point (i.e., failure of (1.4) to hold true) did not cause any numerical instabilities in our test runs.

Higher order rate for $\mathbf{y}_{\mathbf{h}}^{\gamma}$. From the results depicted in Figure 4.4 we observe that the convergence of $\left\|y-y_{h}^{\gamma}\right\|_{L^{2}}$ is (almost) of the order $\mathcal{O}\left(\gamma^{-1}\right)$. This aspect is not covered by the theory of sections 2 and 3 . Rather we offer the following explanation: In [9] it was shown that, under the assumption that the set

$$
S_{0}^{\gamma}:=\{x \in \Omega: \bar{\lambda}(x)+\gamma y(x)=0\}
$$



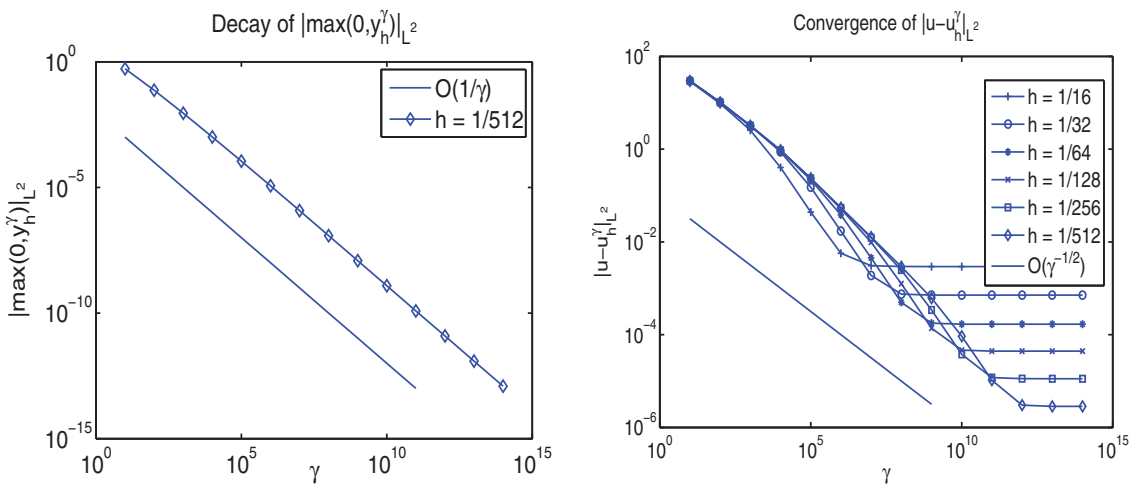

Fig. 4.3. Example 2. Decay of $\left\|\left(y_{h}^{\gamma}\right)^{+}\right\|_{L^{2}}$ (left) and the convergence of $\left\|u-u_{h}^{\gamma}\right\|_{U}$ (right).
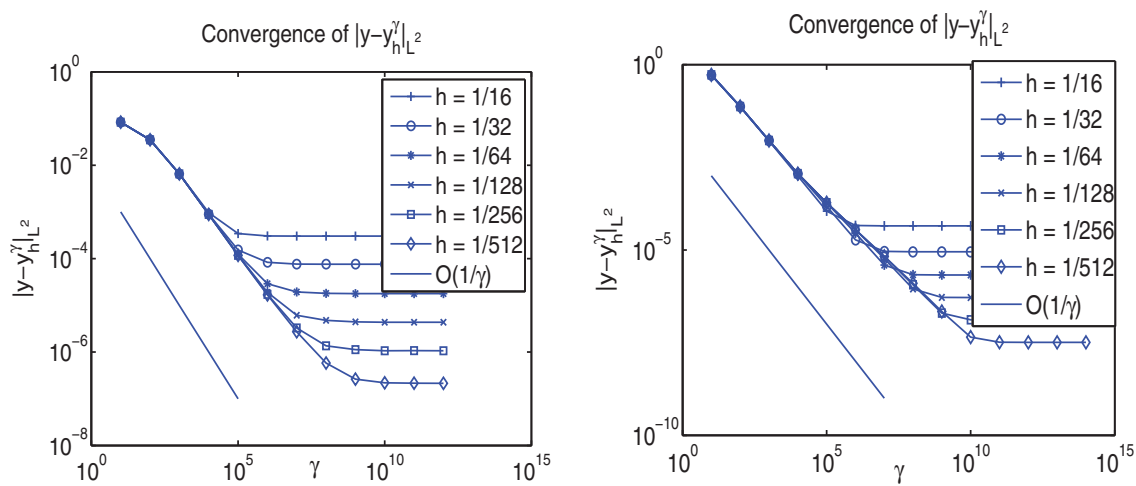

Fig. 4.4. Convergence of $\left\|y-y_{h}^{\gamma}\right\|_{L^{2}}$ for Example 1 (left) and Example 2 (right).

has measure zero, $y^{\gamma}$ and $u^{\gamma}$ (and, hence, $p^{\gamma}$ ) are strongly differentiable (in $H^{2}(\Omega) \cap$ $H_{0}^{1}(\Omega)$ and $L^{2}(\Omega)$ ) with respect to $\gamma$. Let $\dot{y}^{\gamma}$ and $\dot{u}^{\gamma}$ denote the corresponding derivatives. We point out that in what follows we argue only for homogeneous Dirichlet boundary conditions as this reflects our numerical example. Other boundary conditions (such as the Neumann condition discussed earlier) can be covered with little modification. In [9] it was further shown that the above derivatives satisfy a system of sensitivity equations (see Proposition 2.4 and Corollary 2.1 in [9]):

$$
\begin{aligned}
-\Delta \dot{y}^{\gamma}-\dot{u}^{\gamma} & =0, \\
-\Delta \dot{p}^{\gamma}-\chi_{S^{\gamma}}\left(y^{\gamma}+\gamma \dot{y}^{\gamma}\right)-\dot{y}^{\gamma} & =0, \\
\alpha \dot{u}^{\gamma}+\dot{p}^{\gamma} & =0,
\end{aligned}
$$

where $S^{\gamma}:=\left\{x \in \Omega: \bar{\lambda}(x)+\gamma y^{\gamma}(x)>0\right\}$ and $\chi_{S}$ denotes the characteristic function of a set $S \subset \Omega$. Replacing $\dot{p}^{\gamma}$ by $-\alpha \dot{u}^{\gamma}$, reducing the remaining two equations to one, and testing with $\dot{y}^{\gamma} \in H^{2}(\Omega) \cap H_{0}^{1}(\Omega)$ results in

$$
\alpha\left\|\Delta \dot{y}^{\gamma}\right\|_{L^{2}}^{2}+\left\|\dot{y}^{\gamma}\right\|_{L^{2}}^{2}+\gamma\left\|\dot{y}^{\gamma}\right\|_{L^{2}\left(S^{\gamma}\right)}^{2}=\left(\chi_{S^{\gamma}} y^{\gamma}, \dot{y}^{\gamma}\right) .
$$

Copyright (C) by SIAM. Unauthorized reproduction of this article is prohibited. 
Assuming $\left\|\left(y^{\gamma}\right)^{+}\right\|_{L^{2}}=\mathcal{O}(\kappa(\gamma))$ with a continuous function $\kappa$ satisfying $\kappa(\gamma) \downarrow 0$ as $\gamma \rightarrow \infty$, from (4.6) we immediately derive

$$
\begin{aligned}
\left\|\dot{y}^{\gamma}\right\|_{L^{2}} & \leq\left\|\left(y^{\gamma}\right)^{+}\right\|_{L^{2}}=\mathcal{O}(\kappa(\gamma)) \\
\left\|\Delta \dot{y}^{\gamma}\right\|_{L^{2}}^{2} & \leq \alpha^{-1}\left\|\dot{y}^{\gamma}\right\|_{L^{2}}\left\|\left(y^{\gamma}\right)^{+}\right\|_{L^{2}}=\mathcal{O}\left(\kappa(\gamma)^{2}\right), \\
\left\|\dot{y}^{\gamma}\right\|_{L^{2}\left(S^{\gamma}\right)} & \leq \gamma^{-1}\left\|\left(y^{\gamma}\right)^{+}\right\|_{L^{2}}=\mathcal{O}\left(\gamma^{-1} \kappa(\gamma)\right) .
\end{aligned}
$$

Note that we (at least) have $\kappa(\gamma)=\gamma^{-1 / 2}$. In our numerical example we rather observe $\kappa(\gamma)=\gamma^{-1}$ (see also Theorems 2.2 and 2.3 for theoretical investigations). Now, assuming that when increasing $\gamma$ and $y^{\gamma}$ changes most in the region where it violates the pointwise inequality constraint $y \leq 0$, we invoke the assumption

$$
\left\|\dot{y}^{\gamma}\right\|_{L^{2}\left(S_{c}^{\gamma}\right)} \leq C\left\|\dot{y}^{\gamma}\right\|_{L^{2}\left(S^{\gamma}\right)}
$$

for some positive constant $C$ independent of $\gamma$. Above $S_{c}^{\gamma}$ denotes the complement of $S^{\gamma}$ in $\Omega$. We point out that we could weaken (4.10) by allowing some dependence of $C$ on $\gamma$ as long as $C \kappa(\gamma)=\mathcal{O}\left(\gamma^{-t}\right)$ for some $t>0$. But, for the sake of simplicity, we keep the $\gamma$-independent formulation. Then, still assuming that the $d$-dimensional Lebesque measure of $S_{0}^{\gamma}$ vanishes for all $\gamma>0$, we obtain

$$
\left\|y^{\gamma}-y\right\|_{L^{2}} \leq \lim _{\tau \rightarrow \infty} \int_{\gamma}^{\tau}\left\|\dot{y}^{s}\right\|_{L^{2}} d s \leq \hat{C} \lim _{\tau \rightarrow \infty} \int_{\gamma}^{\tau} s^{-1} \kappa(\gamma) d s,
$$

with some positive constant $\hat{C}$ depending on $C$. In the case where $\kappa(\gamma)=\gamma^{-1 / 2}$, we obtain

$$
\left\|y^{\gamma}-y\right\|_{L^{2}}=\mathcal{O}\left(\gamma^{-1 / 2}\right)
$$

and

$$
\left\|y^{\gamma}-y\right\|_{L^{2}}=\mathcal{O}\left(\gamma^{-1}\right)
$$

for $\kappa(\gamma)=\gamma^{-1}$. The latter case appears to be reflected in Figure 4.4.

Conclusions. In this paper we develop error estimates for the Moreau-Yosida regularization of state constrained optimal control problems. A critical tool is an estimate for the pointwise violation of the constraint due to the involved relaxation in $L^{2}(\Omega)$. Based on this result and an estimate for the finite element discretization error, an estimate for the overall error is obtained which allows an optimal regularization parameter adjustment. The theoretical findings are verified numerically. Further, based on our numerical results, we also argue estimates for the state in the $L^{2}$-norm. Our second numerical example shows that, even in cases where the Slater condition fails to hold, our error estimates seem to remain true. A thorough analytical investigation of this situation under a weaker constraint qualification remains subject to future work.

\section{Appendix A. Projection.}

Lemma A.1. Let $(X,(\cdot, \cdot))$ denote a real Hilbert space, $S \subseteq X$ a convex and closed subset, and $P: X \rightarrow S$ the orthogonal projection in $X$ onto $S$. Then

$$
\|P(s)-P(t)\|^{2} \leq(s-t, P(s)-P(t)) \text { for all } s, t \in X .
$$


Proof. $P: X \rightarrow S$ is the orthogonal projection in $X$ onto $S$ iff

$$
(a-P(a), b-P(a)) \leq 0 \text { for all } b \in S .
$$

Use now $a=s, b=P(t)$ and $a=t, b=P(s)$, and add the resulting inequalities. This proves the assertion.

We have the following immediate consequence of Lemma A.1.

Corollary A.2. In Lemma A.1, let $X:=L^{2}(\Omega)$ and $S:=\{y \geq 0$ a.e. in $\Omega\}$. Then for $y \in L^{2}(\Omega)$

$$
P(y)(x)=y^{+}(x):=\max (0, y(x)) \text { a.e. in } \Omega .
$$

\section{REFERENCES}

[1] E. CASAS, $L^{2}$ estimates for the finite element method for the Dirichlet problem with singular data, Numer. Math., 47 (1985), pp. 627-632.

[2] S. Brenner and R. Scott, The Mathematical Theory of Finite Elements, 2nd ed., Springer, New York, 2002.

[3] E. CASAS, Control of an elliptic problem with pointwise state constraints, SIAM J. Control Optim., 24 (1986), pp. 1309-1318.

[4] J. Douglas, T. Dupont, And L. Wahlbin, The stability in $L^{q}$ of the $L^{2}$-projection into finite element function spaces, Numer. Math., 23 (1975), pp. 193-197.

[5] K. Deckelnick And M. Hinze, Convergence of a finite element approximation to a stateconstrained elliptic control problem, SIAM J. Numer. Anal., 45 (2007), pp. 1937-1953.

[6] K. Deckelnick and M. Hinze, A Finite Element Approximation to Elliptic Control Problems in the Presence of Control and State Constraints, Preprint HBAM2007-01, Hamburger Beiträge zur Angewandten Mathematik, Universität Hamburg, Hamburg, Germany, 2007.

[7] K. Deckelnick And M. Hinze, Numerical analysis of a control and state constrained elliptic control problem with piecewise constant control approximations, Proceedings of ENUMATH 2007, Numerical Mathematics and Advanced Applications, Springer, Berlin, 2008, pp. 597-604.

[8] M. Hintermüller and K. Kunisch, Path-following methods for a class of constrained minimization problems in function space, SIAM J. Optim., 17 (2006), pp. 159-187.

[9] M. Hintermüller And K. Kunisch, Feasible and noninterior path-following in constrained minimization with low multiplier regularity, SIAM J. Control Optim., 45 (2006), pp. 11981221.

[10] M. HintermüLler, Mesh-independence and fast local convergence of a primal-dual activeset method for mixed control-state constrained elliptic problems, ANZIAM J., 49 (2007), pp. $1-38$.

[11] M. Hinze, A variational discretization concept in control constrained optimization: The linearquadratic case, Comput. Optim. Appl., 30 (2005), pp. 45-61.

[12] M. Hinze And C. Meyer, Variational discretization of Lavrentiev-regularized state constrained elliptic optimal control problems, Comput. Optim. Appl., 1 (2008).

[13] M. Hinze And A. Schiela, Discretization of Interior Point Methods for State Constrained Elliptic Optimal Control Problems: Optimal Error Estimates and Parameter Adjustment, Preprint SPP1253-08-03, German Research Foundation, Priority Programme 1253, Universität Erlangen, 2007.

[14] R. Scотт, Optimal $L^{\infty}$ estimates for the finite element method on irregular meshes, Math. Comp., 30 (1976), pp. 681-897.

Copyright (c) by SIAM. Unauthorized reproduction of this article is prohibited. 